\title{
A space-time geostatistical approach for ensemble rainfall nowcasting
}

\author{
A. CASERI ${ }^{1, a}$, M.-H. RAMOS ${ }^{2}$, P. JAVELLE ${ }^{1}$ and E. LEBLOIS ${ }^{3}$ \\ ${ }^{1}$ IRSTEA, UR OHAX, Aix-en-Provence, France \\ 2 IRSTEA, UR HBAN, Antony, France \\ ${ }^{3}$ IRSTEA, UR HHLY, Lyon, France
}

\begin{abstract}
Nowcasting systems are essential to prevent extreme events and reduce their socio-economic impacts. The major challenge of these systems is to capture high-risk situations in advance, with good accuracy, location and time. Uncertainties associated with the precipitation events have an impact on the hydrological forecasts, especially when it concerns localized flash flood events. Radar monitoring can help to detect the space-time evolution of rain fields, but nowcasting techniques are needed to go beyond the observation and provide scenarios of rainfall for the next hours of the event. In this study, we investigate a space-time geostatistical framework to generate multiple scenarios of future rainfall. The rainfall ensemble is generated based on space-time properties of precipitation fields given by radar measurements and rainfall data from rain gauges. The aim of this study is to investigate the potential of a framework that applies a geostatistical conditional simulation method to generate an ensemble nowcasting of rainfall fields. The Var region (south eastern France) and 14 events are used to validate the approach. Results show that the proposed method can be a solution to combine information from radar fields and rain gauges to generate nowcasting rainfall fields adapted for flash flood alert.
\end{abstract}

\section{Introduction}

Flash floods are responsible for economic and social losses around the world. Predicting these events is considered one of the hardest and most challenging situations faced by hydrologists and meteorologists [1]. In France, flash floods are also natural hazards of high socioeconomic impact [2]. The event of 3 October 2015, which took place in the south of France, caused several damages and a total of twenty casualties. The "Alpes-Maritimes" French "department" was one of the regions most affected area. For instance, in Cannes, an exceptional point rainfall intensity of $175 \mathrm{~mm}$ was observed in 2 hours ([3], [4]).

The tools available to improve the anticipation of flash floods include the measurement and monitoring of rainfall events by weather radars with high spatial and time resolution (eg., [5]) and the development of high resolution numerical weather prediction models (eg., [6]; [7]). Beyond the diversity of approaches and applications, quantifying the meteorological and hydrological uncertainties for nowcasting is a challenge. Notably, uncertainties associated with the rainfall events have an important impact on the hydrological forecasts, especially when it concerns localized flash flood events.

In hydrometeorological forecasting, ensemble prediction is an approach increasingly used to quantify predictive uncertainties and to improve the reliability of forecasts. An example of short-term prediction ensemble method is the STEPS (Short Term Prediction Systems) approach ([7]; [8]). Based on a combination of extrapolation, perturbation and cascading of nowcasting weather predictions, it allows the forecast of an event with a lead time of up to 6 hours ahead. Another example is the SBMcast method ([9]). Based on a Lagrangian extrapolation of the latest observed rainfall radar field, it generates rainfall scenarios that preserve the spatial and temporal structure of the observed fields.
In France, ensemble weather forecasts have also been investigated for short-term flood forecasting (up to about 72 hours), based on the ARPEGE (PEARP) meteorological model ([10]; [11])

In this study, we investigate a space-time geostatistical framework to generate multiple scenarios of future rainfall for the nowcasting of flash flood events. The geostatistical approach is based on a conditional simulation and an advection method applied within the Turning Bands Method (TBM). Ensemble rainfall nowcasts are generated based on space-time properties of precipitation fields given by radar measurements and precipitation data from rain gauges. The approach has been previously tested for the simulation of uncertainty in radar rainfall fields ([12]) and is here proposed for nowcasting. The method was applied in the Var region, south of France, using several flood events.

This paper is organized as follows: section 2 describes the development of the method and the methodology of evaluation of the results; section 3 describes the study area and the data; section 4 presents the results, and section 5 draws the conclusions.

\section{Method to generate ensembles}

\subsection{The TBM method to generate precipitation ensembles}

The precipitation ensembles are generated using a geostatistical simulation method, the SAMPO Turning Band Method (TBM) developed at Irstea. The algorithm simulates rain in space $(\mathrm{x}, \mathrm{y})$ and time $(\mathrm{z})$ using a space-time variogram model and an advection technique. The advection incorporates a velocity and is considered uniform and constant over the duration of an event. Further details about the generator can be found in [13] and references therein. We adapted the SAMPO TBM method to perform

a Corresponding author: angelica.caseri@irstea.fr

(C) The Authors, published by EDP Sciences. This is an open access article distributed under the terms of the Creative Commons Attribution 
conditional simulation, following the first tests reported in [14]. Basically, the framework consists of a non-conditional TBM simulation of random fields in space and time, followed by a conditioning step at rain gauge locations.

At the points of conditioning, all simulated fields have the same value as the data. Conditioning is based on the residual substitution kriging approach and MCMC sampling (further details can be found in [12]). In summary, the parameters used by the generator are: the wind velocity and direction of the event, the space-time variogram model, the average percentage of zero rain, the direction and velocity of rain cells, the mean and standard deviation of the precipitation data, and the rain data at gauged locations for the conditioning.

In nowcasting, the parameters of the generator are estimated considering the rainfall data available at the time of the start of the nowcasting. The process has to start after a minimum of hours after the start of an event. Here, we consider 4 hours for this initialization. The rainfall nowcasts are then issued for the next six hours, with a total of 30 ensemble members generated at each hourly time step of the event. For the subsequent time steps, we used an increasing window approach: at each new start of a nowcast, we recalculate the parameters of the generator using all available data since the beginning of the event. This is repeated at each hourly time step and up to the end of the event. Figure 1 illustrates the schema adopted in this study.

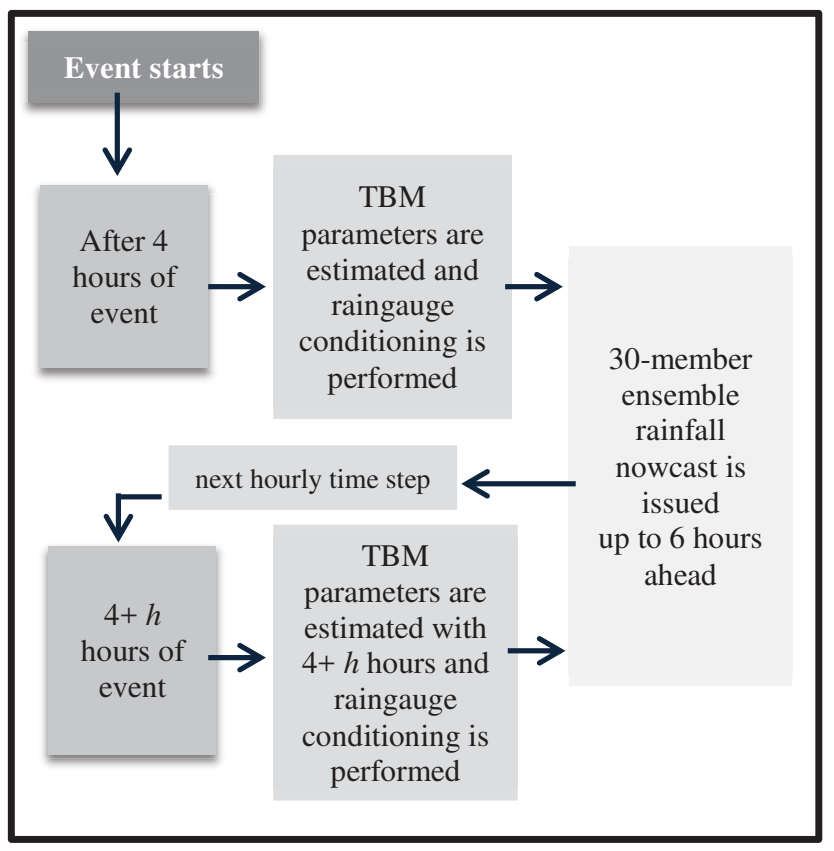

Figure 1. Schema adopted for the generation of ensemble rainfall nowcasts: the time step loop is repeated up to the end of the event.

\subsection{Evaluation}

We compared the rain fields generated with the SAMPO TBM method with an independent rainfall dataset provided by Météo-France, which is a merge of radar imagery and rain gauges measurements (ANTILOPE product). All events were considered together to calculate statistical scores over all the time steps available. The rainfall nowcasts were evaluated for each lead time, either considering each rain pixel of the study area (maps of scores) or areal rainfalls over selected catchments.
The accuracy of the ensemble nowcasts was evaluated considering the ensemble mean and the Mean Absolute Error (MAE). MAE values range from 0 to $\infty$ and are negatively-oriented scores, i.e., lower values are better. We also computed the Critical Success Index (CSI), the Probability of detection (POD) and the Success Ratio (SR), for which we considered a probability threshold of at least $20 \%$ of the ensemble members exceeding a precipitation threshold of $1 \mathrm{~mm} / \mathrm{h}$. For these criteria, the value of 1 represents a "perfect prediction", where there are no false alarms or missed alerts [9]. Scores maps were generated for these criteria.

We also calculated the Relative Operating Characteristic (ROC). It measures the ability of predictions to discriminate between events and non-events. It is widely used in the evaluation of probabilistic forecasts and is considered as a measure of potential usefulness. It is characterized by the graphical representation of the probability of detection (POD) against the probability of false detection (POFD), using different predictive probability thresholds (eg, at least $5 \%, 25 \%, 50 \%$, etc. of ensemble members exceeding a given threshold). The area under the ROC (AUC) can be computed to estimate the quality of the forecast datasets. A perfect prediction is represented by an AUC equal to 1, while an AUC below 0.5 indicates a prediction with no skill (not better than an aleatory forecast). The ROC was applied to evaluate rainfall nowcasts at the catchment scale. For this, a threshold was considered, which corresponds to the $70 \%$ quantile of areal rainfall, defined at each studied catchment.

More details on the evaluation scores used in this study can be found in [15].

\section{Case-study and data}

The methodology was applied to the Var "Département" in the French Mediterranean region. It is indicated in Fig. 2, together with the rain gauge network and the studied catchments.

The data used to generate precipitation ensembles come from 30 rain gauges and the PANTHERE weather radar data, which is the French operational radar data that provides quantitative precipitation fields in real time. It consists of 5-min precipitation fields with a $1 \times 1 \mathrm{~km}^{2}$ grid resolution. To evaluate the rainfall nowcasts, we compared them against the ANTILOPE radar data. This is a hybrid product, coming from the PANTHERE data and a rain gauge interpolation (kriging with external drift). All meteorological data were provided by the French meteorological service (Météo-France). In our study, these data were aggregated at the hourly time step. The data span from October 2009 to March 2013, with the presence of some missing data values at the rain gauge locations.

A total of 22 catchments of different area sizes were selected for this study (Fig. 2). These catchments were used to calculate the catchment area rainfall (observed and nowcast rainfall). From the data available, we selected 14 events.

Each event was considered to start (or to end) when at least one (or no) hourly radar pixel exceeded $5 \mathrm{~mm} / \mathrm{h}$ anywhere in the study region. Only the most significant events in terms of rainfall accumulation and spatial extension were considered. The selected events have the following characteristics:

- The duration of the events range from 8 to 34 hours. 
- The maximum observed rainfall at a radar pixel varies between $13 \mathrm{~mm} / \mathrm{h}$ and $92 \mathrm{~mm} / \mathrm{h}$.

- The maximum accumulated rainfall value (over the total duration of the event), observed at a radar pixel, ranges from $53 \mathrm{~mm}$ to $414 \mathrm{~mm}$.

- The majority of the events occur during the fall and winter seasons, but one of the most intense events was observed in June, during summer 2010. This event lasted 31 hours, with a total point rainfall of $357 \mathrm{~mm}$ and a maximum hourly rainfall of $92.4 \mathrm{~mm}$ at a radar pixel.

- The majority of the events had higher intensities falling in the eastern part of the Var region.

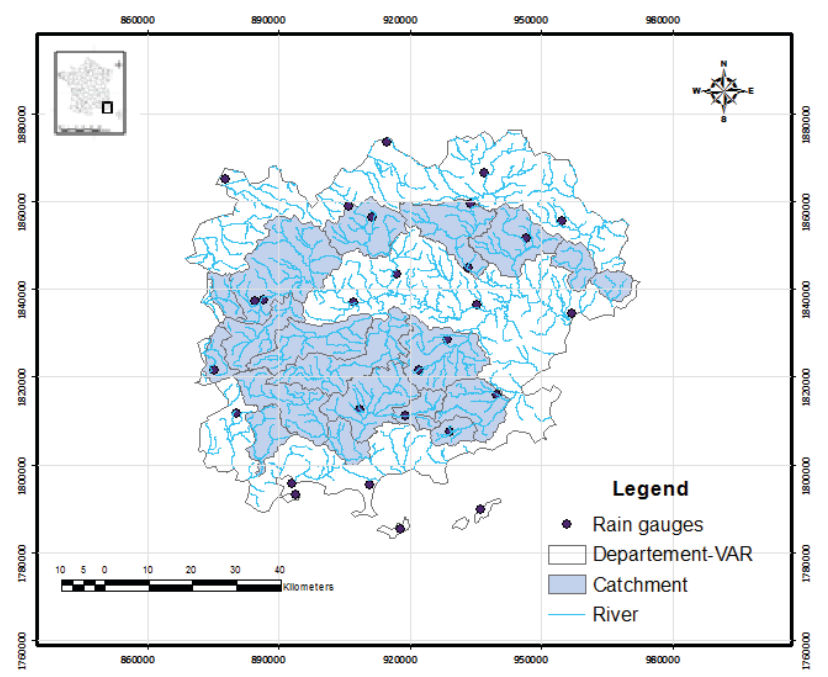

Figure 2. Location of the study area (Var region) in southeast France and its river network. Studied catchments are coloured in blue and rain gauges are indicated by dots.

\section{Results}

\subsection{Parameters of the rainfall generator}

Parameters of the TBM algorithm were evaluated separately for each rain event and considering the last observed hourly time steps. They were used to generate the rainfall nowcasts. Hourly weather radar data were used to fit an exponential model to the hourly empirical space-time variograms. The spatial and temporal variogram of the precipitation intermittency has a range between $21-77 \mathrm{~km}$ in space and 2$5 \mathrm{~h}$ in time. The nonzero precipitation variograms present a spatial range between $13-89 \mathrm{~km}$ and a temporal range between 1-7 h. The average percentage of zero precipitation, varies between $5 \%$ and $51 \%$ among the studied events. For each event, the wind velocity and the direction were estimated. Values vary between $2.5 \mathrm{~m} / \mathrm{s}$ and $13 \mathrm{~m} / \mathrm{s}$, and the majority of the events show wind direction from southeast to northwest. Rain gauge data were used to parameterise the cumulative distribution function (CDF). The mean and the standard deviation of nonzero rain vary between $1-4.6 \mathrm{~mm} / \mathrm{h}$ and $0.7-10 \mathrm{~mm} / \mathrm{h}$, respectively.

\subsection{Evaluation of the rainfall nowcasting with score maps}

The forecasts generated by the SAMPO TBM method (increasing window) were evaluated against observed rainfalls and score maps were obtained. Score maps were also computed for a nowcast consisting of a persistent rainfall, i.e., the last observed rainfall radar field is considered as the rainfall nowcast for the six hours ahead.

Figure 3 presents the MAE maps obtained for lead times from one hour to four hours. MAE values were computed for each precipitation pixel $\left(1 \mathrm{~km}^{2}\right)$. We can see that the MAE values increase as lead time increases. There is an important difference between the MAE obtained using the SAMPO TBM method and the MAE from the persistence method. The latter can reach up to $5 \mathrm{~mm} / \mathrm{h}$, while the former has much lower values, mainly after the first hour ahead. We also observe that MAE values are higher in the eastern part of the study area. This is due to the fact that this is the rainiest area in the region, with several events in our dataset registering high rain values over this area.

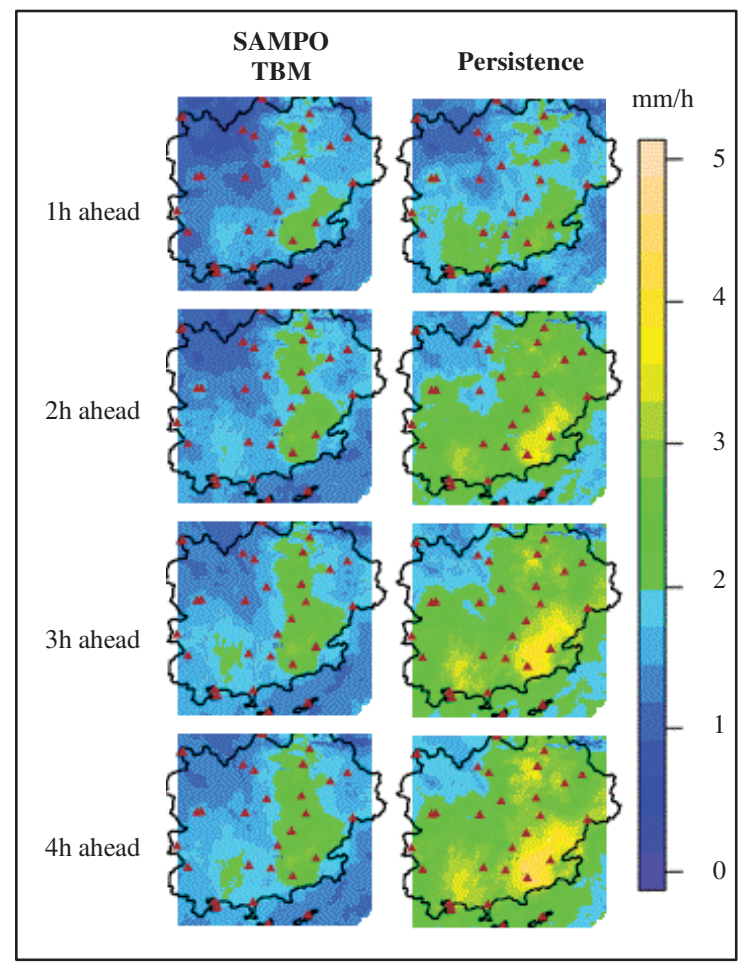

Figure 3. MAE maps calculated considering the ensemble mean forecast generated using the SAMPO TBM method (left) and the persistence method (right). The red triangles represent the rain gauges.

Figure 4 presents the CSI, POD and SR maps. We can see that the scores are better at the first hours ahead and the nowcasts tend to lose performance as lead time increases. Also here, in general, the nowcasts obtained with the rainfall generator show better performance than the persistence method, notably after the first hour ahead. 


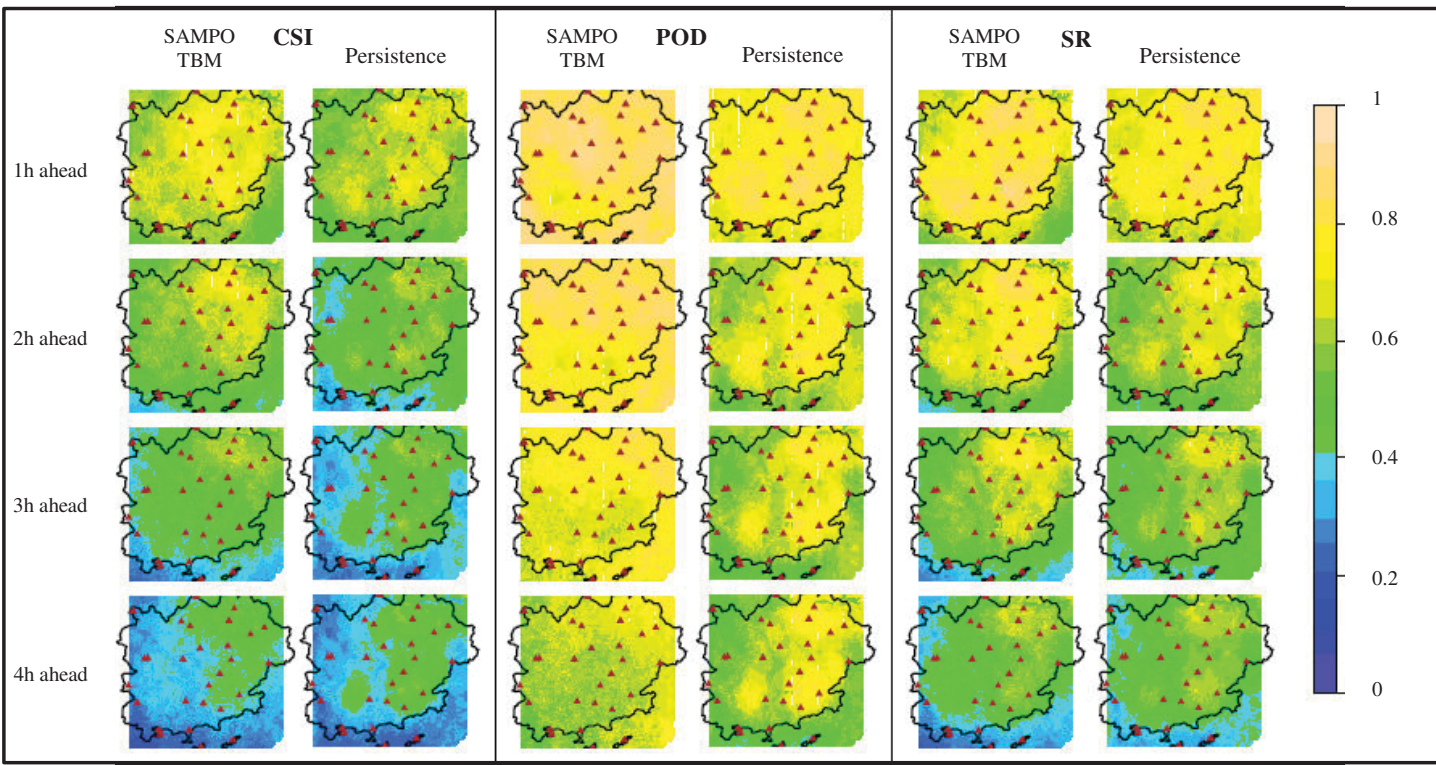

Figure 4. CSI (left), POD (centre) and SR (right) maps for the SAMPO TBM method (SAMPO TBM) and the persistence method. The red triangles represent the rain gauges.

\subsection{Evaluation of the rainfall nowcasting over the studied catchment areas}

Figure 5 presents the ROC AUC obtained for the studied catchments and considering all the studied events. It can be observed that the AUC also decreases when lead time increases, as observed with the other evaluation scores. The AUC is below 0.5, meaning that the prediction has no skill. After 3 hours, its performance decreases significantly. This can be related to the ranges of correlation of the space-time variogram. The average of the time correlation range over the studied events is about three hours. The SAMPO TBM method can thus generate rain cells in places where actually there is no rain. The probability of false detection (POFD) will increase and, consequently, there is a reduction of the ROC AUC.

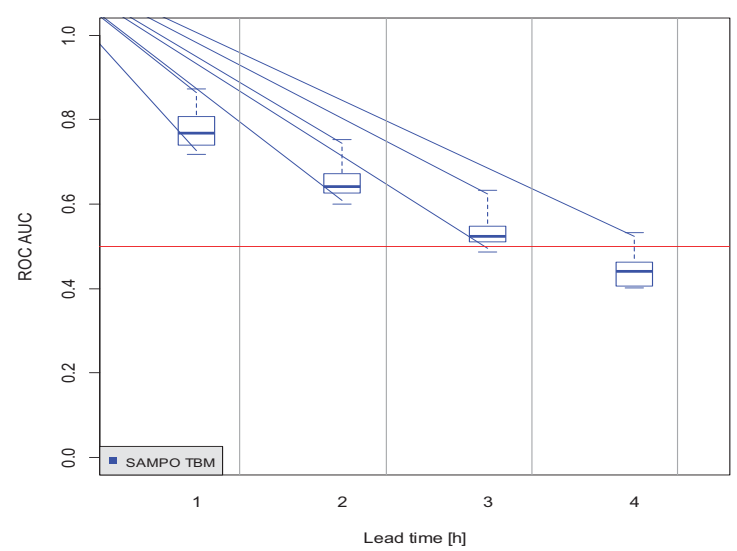

Figure 5. Variation of the ROC AUC obtained at 22 catchments according to lead time. Rainfall nowcasts from the SAMPO TBM method (blue) is shown. The red line represents the 0.5 value, below which predictions have no skill. The boxplots represent the $5 \%, 25 \%, 50 \%, 75 \%$ and $95 \%$ quantile.

\section{Conclusion}

In this paper, we investigated a geostatistical framework method to generate space-time rainfall ensembles for nowcasting, using both rainfall radar data and rain gauge data. The methodology was applied to 14 rain events observed in the Var region in France and evaluated over 22 catchments.

The results indicate that the approach set up in this study was able to simulate an ensemble of rainfall forecast fields with, in general, better performance for the next hours ahead than a persistence-based method. The ensemble nowcasting of rainfall fields can be used as input to a hydrological model and, ultimately, provide information on the probability of exceeding critical flood alert thresholds. Future work will explore these challenges.

\section{References}

[1] Meon G. (2006). Past and present challenges in flash flood forecasting. First International Workshop on Flash Flood Forecasting, Costa Rica, 2.

[2] Bastone V., De la Torre Y. and Garnier C. H. (2011). Etude préliminaire de l'impact du changement climatique sur les risques naturels à la Réunion. Raport BRGM/RP-59495-FR, 135.

[3] Javelle P., Organde, D., Saint-Martin, C., Demargne, J., Saint-Aubin, C., Garandeau, L., Janet, B. (2016). Setting up a French national flash flood warning system for ungauged catchments based on the AIGA method. FLOODrisk 2016 - $3^{\text {rd }}$ European Conference on Flood Risk Management, Lyon, 17-21 October 2016, in this issue.

[4] Saint-Martin C., Fouchier C., Javelle P., Douvinet J., and Vinet F. (2016). Assessing the exposure to floods to estimate the risk of flood-related damage in French Mediterranean basins. FLOODrisk 2016 - $3^{\text {rd }}$ European Conference on Flood Risk Management, Lyon, 17-21 October 2016, in this issue. 
[5] Smith J. A., Baeck M. L., Meierdiercks K. L., Miller A. J., and Krajewski W. F. (2007). Radar rainfall estimation for flash flood forecasting in small urban watersheds. Advances in Water Resources, 30, 2087 2097.

[6] Vincendon B., Ducrocq V., Nuissier O., and Vié B. (2011). Perturbation of convection-permitting NWP forecasts for flash-flood ensemble forecasting. Natural Hazards Earth System Sciences, 11(5), 15291544.

[7] Bowler N. E., Pierce C. E., and Seed A. W. (2006). STEPS: A probabilistic precipitation forecasting scheme which merges an extrapolation nowcast with downscaled NWP. Quarterly Journal of the Royal Meteorological Society, 132, 2127-2155.

[8] Collier C. G. (2007). Flash flood forecasting: What are the limits of predictability? Quarterly Journal of the Royal Meteorological Society, 133, 3-23.

[9] Berenguer M., Sempere-Torres D., and Pegram G. G. S. (2011). SBMcast - An ensemble nowcasting technique to assess the uncertainty in rainfall forecasts by Lagrangian extrapolation. Journal of Hydrology, 404, 226-240.

[10] Randrianasolo A., Ramos M. H., Thirel G., Andréassian V., and Martin E. (2010). Comparing the scores of hydrological ensemble forecasts issued by two different hydrological models," Atmospheric Science Letters, 11(2), 100-107.

[11] Bourgin F., Andréassian V., Perrin C., and Oudin L. (2015). Transferring global uncertainty estimates from gauged to ungauged catchments. Hydrology and Earth System Sciences, 19, 2535-2546.

[12] Caseri A., Javelle P., Ramos M. H., and Leblois E. (2015). Generating precipitation ensembles for flood alert and risk management," Journal of Flood Risk Management. DOI: $10.1111 / \mathrm{jfr} 3.12203$.

[13] Leblois E. and Creutin J.-D. (2013). Space-time simulation of intermittent rainfall with prescribed advection field: Adaptation of the turning band method Water Resources Research, 49, 3375-3387.

[14] Renard B., Kavetski D., Leblois E., Thyer M., Kuczera G. and Franks S. W. (2011). Toward a reliable decomposition of predictive uncertainty in hydrological modeling: Characterizing rainfall errors using conditional simulation. Water Resources Research, 47 (11). DOI: 10.1029/2011WR010643.

[15] Jolliffe I. T. and Stephenson D. B. (2011). Forecast Verification: A Practitioner's Guide in Atmospheric Science. 2nd ed. Wiley, 292. 